\title{
CORRIGENDUM
}

\section{Physical interaction between peroxisomes and chloroplasts elucidated by in situ laser analysis}

Kazusato Oikawa, Shigeru Matsunaga, Shoji Mano, Maki Kondo, Kenji Yamada, Makoto Hayashi, Takatoshi Kagawa, Akeo Kadota, Wataru Sakamoto, Shoichi Higashi, Masakatsu Watanabe, Toshiaki Mitsui, Akinori Shigemasa, Takanori lino, Yoichiroh Hosokawa and Mikio Nishimura

Nature Plants 1, 15035 (2015); published online 30 March 2015; corrected online 10 April 2015.

In the version of the Supplementary Information file originally published figure captions were omitted and several characters in the text were rendered incorrectly. These errors have been corrected in this file 10 April 2015. 